\title{
Fuzzy Soft Topology Based on Generalized Intersection and Generalized Union of Fuzzy Soft Sets
}

\author{
Jingshui Ping ${ }^{1}$ and Guangming Xue $\mathbb{D}^{2}$ \\ ${ }^{1}$ School of Finance and Mathematics, Huainan Normal University, Huainan 232038, China \\ ${ }^{2}$ School of Information and Statistics, Guangxi University of Finance and Economics, Nanning 530003, China \\ Correspondence should be addressed to Guangming Xue; xueguangming0417@126.com
}

Received 22 August 2021; Revised 24 October 2021; Accepted 28 October 2021; Published 15 November 2021

Academic Editor: Peijun Wang

Copyright ( $) 2021$ Jingshui Ping and Guangming Xue. This is an open access article distributed under the Creative Commons Attribution License, which permits unrestricted use, distribution, and reproduction in any medium, provided the original work is properly cited.

\begin{abstract}
In this study, the generalized intersection and union operations of fuzzy soft set (FSS) are established on the basis of traditional FSS operations, which overcome the shortcomings of traditional FSS operations that do not meet De Morgan's law, and a series of properties of generalized intersection and union operations of FSS are obtained. The fuzzy soft topology under generalized intersection and generalized union operation of FSSs is established. Finally, the topological construction of weak FSS and strong FSS is discussed, and the relationship between them and the topological construction of traditional FSS is obtained.
\end{abstract}

\section{Introduction}

In real life, the data of many complex problems often have uncertainty, randomness, and fuzziness. Probability theory, fuzzy sets [1], intuitionistic fuzzy sets [2], rough sets [3], and so on are the main methods to deal with uncertain data, obtained in various fields of engineering, computer science, economics, medical science, etc., but these theories have their own difficulties, mainly reflected in the lack of parameter tools. In 1999, Molodtsov established soft set theory [4], which overcame the deficiency of parameter tools of these methods and was successfully applied to decision analysis, pattern recognition, data mining, and other fields [5-7], attracting the attention of scholars in many fields [8-13]. In 2021, Guilong Liu outlines a possible relationship between knowledge structures and rough sets [14]. Swarup $\mathrm{Kr}$ Ghosh et al. represent a colonogram enhancement approach using intuitionistic fuzzy set [15]. In 2001, Liu et al. combined the fuzzy set with soft set and proposed the concept of fuzzy soft set (FSS) [11]. Then, Das et al. redefined the concept of FSS and studied its operation. In 2011, Bekir Tanay and M. Burc Kandemir established the topological structure of fuzzy soft sets based on some basic definitions in the topological space of Munkres point sets and combined with the operational properties of FSSs [13]. In addition, Ayetan and Cemil gave the separation axiom and fuzzy soft connectivity of fuzzy soft topological space. In 2018, Ayten and Cemil established mixed fuzzy soft topological spaces [16]. Finally, Riaz et al. gave a bipolar fuzzy soft topology with decision [17]. In 2020, José Carlos R. Alcantud established soft open bases and a novel construction of soft topologies from bases for topologies [18]. By 2021, Ultami and Haripamyu have systematically studied the application of intuitional fuzzy soft sets in topological structures [19].

The existing intersection and union operations of FSSs do not satisfy De Morgan's law. In the study of fuzzy soft topological space structure and properties, the intersection, union, and complement operations of FSSs need to satisfy De Morgan's law. Considering that De Morgan's law of intersection, union, and complement operations of FSSs is not valid, this paper constructs the generalized intersection and union operations of FSSs on the basis of the existing FSS operations, satisfies De Morgan's law, and further studies the operation properties of the generalized intersection and union operations and constructs the corresponding fuzzy soft topology. Based on the concepts of weak fuzzy soft set 
and strong fuzzy soft set proposed by F.B.Hua and Y.P.Wang in [20], the corresponding structures and properties of fuzzy soft topology are studied.

\section{Fuzzy Soft Set and Its Operation Properties}

Definition 1 (see [11]). Let $U$ be the domain and $A$ be the parameter set; $A \subset E, F(U)$ is the whole fuzzy set over $U$; we define $(f, A)$ a fuzzy soft set (FSS) on $U$, where $f: A \longrightarrow$ $F(u)$ is a mapping. The FSS can be expressed as $(f, A)$ $=\left\{a=\left\{u_{f_{a}(u)} \mid u \in U\right\} \mid a \in A\right\}$, where $f_{a}: U \longrightarrow[0,1]$ (see [13]).

Definition 2 (see [11]). Let $A, B \subset E,(f, A),(g, B)$ be FSSs in domain $U$ if $(f, A)$ is a FSS of $(g, B)$ if and only if (1) $A \subset B$ and (2) $\forall c \in A, \forall x \in U, f_{c}(x) \leq g_{c}(x)$. denoted by $(f, A) \widetilde{c}(g, B),(f, A)=(g, B)$ if and only if $(f, A) \widetilde{c}$ $(g, B)$ and $(g, B) \subset(f, A)$.

Definition 3 (see [21]). Let $(f, E)$ a FSS on $U$ if $(f, E)$ is an empty FSS if and only if $\forall e \in E, f_{e}(x)=0, \forall x \in U$, denoted by $\widetilde{\Phi}$.

Remark 1. In general, $(f, A)$ is an empty FSS on $U$, denoted as $\widetilde{\Phi}_{A}$.

Definition 4 (see [21]). Let $(f, E)$ a FSS on $U$ if $(f, E)$ is an absolute FSS if and only if $\forall e \in E, f_{e}(x)=1, \forall x \in U$, denoted by $\widetilde{U}_{E}$.

Definition 5 (see [21]). The remainder of a FSS $(f, A)$ on $U$ is $\left(f^{c}, A\right)$, where $f^{c}: A \longrightarrow F(U), \forall a \in A$, and $f_{a}^{c}(x)=$ $1-f_{a}(x), \forall x \in U$, denoted by $\left(f^{c}, A\right)=(f, A)^{c}$.

Definition 6 (see [21]). Let $(g, C)$ and $(h, D)$ be FSSs on $U$. The union of $(g, C)$ and $(h, D)$ is $(g, C) \widetilde{\cup}(h, D)=(k, B)$, where $B=C \cup D$ :

$$
\forall b \in B, k_{b}(x)= \begin{cases}g_{b}(x), & b \in C-D, \\ h_{b}(x), & b \in D-C, \quad \forall x \in U . \\ g_{b}(x) \vee h_{b}(x), & b \in C \cap D,\end{cases}
$$

Definition 7 (see [21]). Let $(g, C)$ and $(h, D)$ be FSSs on $U$. The intersection of $(g, C)$ and $(h, D)$ is $(g, C) \widetilde{\cap}(h, D)=$ $(k, B)$, where $B=C \cup D, \forall b \in B, \forall x \in U$, and $k_{b}(x)=g_{b}$ $(x) \wedge h_{b}(x)$.

As can be seen from Definition 7, when $c \in A-B$ or $c \in B-A, \forall x \in U$, and $h_{c}(x)$ has no definition, this will cause De Morgan's law to cease to hold, due to the nature of the algebraic structure of FSS and nature of fuzzy soft topological space structure; setup in a certain level will depend on the virtue of De Morgan's law; therefore, it is very meaningful to improve the intersection and union operation of fuzzy soft sets.

\section{Generalization of Union and Intersection Operation of FSS}

Definition 8 Let $(f, A)$ and $(g, B)$ be FSSs on $U$; the generalized union of $(f, A),(g, B)$ is $(f, A) \tilde{\widetilde{U}}(g, B)=(\tilde{f}, A \cup B)$ $\tilde{\cup}(\tilde{g}, A \cup B)=(\widetilde{h}, A \cup B)$, where $\forall x \in U, \forall a \in A \cup B, \tilde{f}_{a}(x)=$ $\left\{\begin{array}{ll}f_{a}(x), & a \in A, \\ 0, & a \in B-A,\end{array} \tilde{g}_{a}(x)= \begin{cases}g_{a}(x), & a \in B, \\ 0, & a \in A-B .\end{cases}\right.$

Remark 2. It is worth noting that because of

$$
\begin{aligned}
\tilde{h}_{a}(x)= & \begin{cases}\tilde{f}_{a}(x), & a \in A-B, \\
\tilde{g}_{a}(x), & a \in B-A, \\
\tilde{f}_{a}(x) \vee \widetilde{g}_{a}(x), & a \in A \cap B\end{cases} \\
& \cdot \begin{cases}f_{a}(x), & a \in A-B, \\
g_{a}(x), & a \in B-A,=h_{a}(x), \\
f_{a}(x) \vee g_{a}(x), & a \in A \cap B\end{cases}
\end{aligned}
$$

there is $(f, A) \widetilde{\widetilde{U}}(g, B)=(f, A) \widetilde{U}(g, B)$.

Definition 9 Let $(f, A)$ and $(g, B)$ be FSSs on $U$; the generalized intersection of $(f, A),(g, B)$ is $(f, A) \tilde{\tilde{n}}(g, B)=(\tilde{f}, A$ $\cup B) \tilde{\cap}(\bar{g}, A \cup B)=(\bar{h}, A \cup B)$, where $\forall x \in U, \forall a \in A \cup B, \bar{f}_{a}$ $(x)=\left\{\begin{array}{ll}f_{a}(x), & a \in A, \\ 0, & a \in B-A,\end{array}\right.$ and $\bar{g}_{a}(x)= \begin{cases}g_{a}(x), & a \in B, \\ 0, & a \in A-B .\end{cases}$

Remark 3. It is worth noting that because of

$$
\begin{aligned}
\bar{h}_{a}(x) & = \begin{cases}\bar{f}_{a}(x) \wedge 0, & a \in A-B, \\
\bar{g}_{a}(x) \wedge 0, & a \in B-A, \\
\bar{f}_{a}(x) \wedge \bar{g}_{a}(x), & a \in A \cap B,\end{cases} \\
& =\left\{\begin{array}{ll}
0, & a \in A-B, \\
0, & a \in B-A, \\
f_{a}(x) \wedge g_{a}(x), & a \in A \cap B,
\end{array}= \begin{cases}h_{a}(x), & a \in A \cap B, \\
0, & \text { otherwise, }\end{cases} \right.
\end{aligned}
$$

there is $(f, A) \widetilde{\widetilde{\cap}}(g, B)=[(f, A) \widetilde{\cap}(g, B)] \widetilde{\cup} \widetilde{\Phi}_{(A \cup B)-(A \cap B)}$.

It can be seen from Definitions 8 and 9 that the generalized union operation of FSS keeps the original union operation of
FSS, while the generalized intersection operation of FSS makes up for the situation, where $\forall x \in U, h_{c}(x)$ is undefined when $c \in A-B$, or $c \in B-A$ is satisfied in Definition 7 . 
Example 1. Let the domain be $U=\left\{h^{1}, h^{2}, h^{3}\right\}$, the parameter set be $A=\left\{e_{1}, e_{2}\right\}$ and $B=\left\{e_{2}, e_{3}\right\} \subset E=\left\{e_{1}, e_{2}, e_{3}\right\}$, and the two FSSs on $U$ be $(f, A)=\left\{e_{1}=\left\{h_{0.2}^{1}, h_{0.3}^{2}, h_{0.7}^{3}\right\}, e_{2}=\right.$ $\left.\left\{h_{0.5}^{1}, h_{0.4}^{2}, h_{0.2}^{3}\right\}\right\}$ :

$$
\begin{aligned}
(g, B) & =\left\{e_{2}=\left\{h_{0.5}^{1}, h_{0.3}^{2}, h_{0.6}^{3}\right\}, e_{3}=\left\{h_{0.2}^{1}, h_{0.3}^{2}, h_{0.5}^{3}\right\}\right\}, \\
(f, A) \widetilde{\widetilde{U}}(g, B) & =\left\{e_{1}=\left\{h_{0.2}^{1}, h_{0.3}^{2}, h_{0.7}^{3}\right\}, e_{2}=\left\{h_{0.5}^{1}, h_{0.4}^{2}, h_{0.6}^{3}\right\}, e_{3}=\left\{h_{0.2}^{1}, h_{0.3}^{2}, h_{0.5}^{3}\right\}\right\}, \\
(f, A) \tilde{\tilde{\cap}}(g, B) & =\left\{e_{1}=\left\{h_{0}^{1}, h_{0}^{2}, h_{0}^{3}\right\}, e_{2}=\left\{h_{0.5}^{1}, h_{0.3}^{2}, h_{0.2}^{3}\right\}, e_{3}=\left\{h_{0}^{1}, h_{0}^{2}, h_{0}^{3}\right\}\right\} .
\end{aligned}
$$

Theorem 1. The generalization of the union and intersection operation of FSSs satisfies the commutative law, associative law, and distributive law; that is, let $(f, A),(g, B)$, and $(h, C)$ be FSSs on $U$; then, there are

(1) $(f, A) \widetilde{\widetilde{U}}(g, B)=(g, B) \widetilde{\widetilde{U}}(f, A)$

(2) $(f, A) \widetilde{\widetilde{\pi}}(g, B)=(g, B) \widetilde{\widetilde{n}}(f, A)$

(3) $(f, A) \tilde{\tilde{U}}[(g, B) \tilde{\tilde{U}}(h, C)]=[(f, A) \tilde{\tilde{U}}(g, B)] \tilde{\tilde{U}}(h, C)$

(4) $(f, A) \tilde{\tilde{n}}[(g, B) \widetilde{\tilde{n}}(h, C)]=[(f, A) \widetilde{\tilde{n}}(g, B)] \tilde{\tilde{n}}(h, C)$
(5) $(f, A) \widetilde{\widetilde{U}}[(g, B) \widetilde{\widetilde{n}}(h, C)]=[(f, A) \widetilde{\widetilde{U}}(g, B)] \widetilde{\widetilde{\Pi}}$ $[(f, A) \widetilde{U}(h, C)]$

(6) $(f, A) \tilde{\tilde{n}}[(g, B) \widetilde{\tilde{U}}(h, C)]=[(f, A) \quad \tilde{\tilde{n}}(g, B)] \tilde{\tilde{U}}[(f$, $A) \tilde{\tilde{\cap}}(h, C)]$

Proof. (1) and (2) are obviously true according to Definitions 8 and 9 , respectively.

From $(3)$, since $(f, A) \widetilde{\widetilde{U}}[(g, B) \widetilde{\widetilde{U}}(h, C)]=(f, A) \tilde{U}[(g$, B) $\widetilde{U}(h, C)]$, so let

$$
\begin{aligned}
(g, B) & \tilde{\cup}(h, C) \\
& =(k, B \cup C), \quad \forall x \in U, \\
k_{t}(x) & = \begin{cases}g_{t}(x), & t \in B-C, \\
h_{t}(x), & t \in C-B, \\
g_{t}(x) \vee h_{t}(x), & t \in B \cap C,\end{cases} \\
(f, A) \tilde{\cup}[(g, B) \tilde{\cup}(h, C)] & (f, A) \tilde{\cup}(k, B \cup C)=(s, A \cup B \cup C), \quad \forall x \in U, \\
s_{t}(x) & = \begin{cases}f_{t}(x), & t \in A-(B \cup C), \\
k_{t}(x), & t \in(B \cup C)-A, \\
f_{t}(x) \vee k_{t}(x), & t \in A \cap(B \cup C) .\end{cases}
\end{aligned}
$$

Due to $(B \cup C)-A=[B-(A \cup C)] \cup[C-(A \cup B)]$

$\cup[(B \cap C)-A]$ and $A \cap(B \cup C)=(A \cap B \cap C) \cup[(A \cap B)$

$-C] \cup[(A \cap C)-B]$, we obtain

$$
s_{t}(x)= \begin{cases}f_{t}(x), & t \in A-(B \cup C), \\ g_{t}(x), & t \in B-(A \cup C), \\ h_{t}(x), & t \in C-(A \cup B), \\ f_{t}(x) \vee g_{t}(x), & t \in(A \cap B)-C, \\ g_{t}(x) \vee h_{t}(x), & t \in(B \cap C)-A, \\ h_{t}(x) \vee f_{t}(x), & t \in(C \cap A)-B, \\ f_{t}(x) \vee g_{t}(x) \vee h_{t}(x), & t \in A \cap B \cap C .\end{cases}
$$


Since $[(f, A) \widetilde{\widetilde{U}}(g, B)] \widetilde{\widetilde{U}}(h, C)=[(f, A) \widetilde{\widetilde{U}}(g, B)] \widetilde{U}$ $(h, C)$, so let

$$
\begin{aligned}
&(f, A) \tilde{\cup}(g, B)\left(k^{\prime}, A \cup B\right), \quad \forall x \in U, \\
& k_{t}^{\prime}(x)= \begin{cases}f_{t}(x), & t \in A-B, \\
g_{t}(x), & t \in B-A, \\
f_{t}(x) \vee g_{t}(x), & t \in A \cap B,\end{cases} \\
& {[(f, A) \tilde{\cup}(g, B)] \tilde{\cup}(h, C) }=\left(k^{\prime}, A \cup B\right) \tilde{\cup}(h, C)=\left(s^{\prime}, A \cup B \cup C\right), \quad \forall x \in U, \\
& s_{t}^{\prime}(x)= \begin{cases}k_{t}^{\prime}(x), & t \in(A \cup B)-C, \\
h_{t}(x), & t \in C-(A \cup B), \\
k_{t}^{\prime}(x) \vee h_{t}(x), & t \in C \cap(A \cup B) .\end{cases}
\end{aligned}
$$

Due to $(A \cup B)-C=[A-(B \cup C)] \cup[B-(A \cup C)]$ $\cup[(A \cap B)-C]$ and $C \cap(A \cup B)=(A \cap B \cap C) \cup[(A \cap$ $C)-B] \cup[(A \cap B)-C]$, we obtain

$$
s_{t}^{\prime}(x)= \begin{cases}f_{t}(x), & t \in A-(B \cup C), \\ g_{t}(x), & t \in B-(A \cup C), \\ h_{t}(x), & t \in C-(A \cup B), \\ f_{t}(x) \vee g_{t}(x), & t \in(A \cap B)-C, \\ g_{t}(x) \vee h_{t}(x), & t \in(B \cap C)-A, \\ h_{t}(x) \vee f_{t}(x), & t \in(C \cap A)-B, \\ f_{t}(x) \vee g_{t}(x) \vee h_{t}(x), & t \in A \cap B \cap C .\end{cases}
$$

Therefore, $s_{t}(x)=s_{t}^{\prime}(x)$, that is, (3) is true.

From (4), let $(g, B) \widetilde{\widetilde{n}}(h, C)=(k, B \cup C), \forall x \in U$; then,

$$
k_{t}(x)=\left\{\begin{array}{ll}
0, & t \in B-C, \\
0, & t \in C-B, \\
g_{t}(x) \wedge h_{t}(x), & t \in B \cap C,
\end{array}= \begin{cases}g_{t}(x) \wedge h_{t}(x), & t \in B \cap C, \\
0, & \text { otherwise, }\end{cases}\right.
$$

$(f, A) \tilde{\tilde{n}}[(g, B) \widetilde{\tilde{n}}(h, C)]=(f, A) \widetilde{\tilde{n}}(k, B \cup C)=(r, A \cup B \cup C)$,

$$
r_{t}(x)=\left\{\begin{array}{ll}
f_{t}(x) \wedge k_{t}(x), & t \in A \cap(B \cap C), \\
0, & \text { otherwise, }
\end{array}= \begin{cases}f_{t}(x) \wedge g_{t}(x) \wedge h_{t}(x), & t \in A \cap(B \cap C) \\
0, & \text { otherwise. }\end{cases}\right.
$$


Let $(f, A) \widetilde{\widetilde{\cap}}(g, B)=\left(k^{\prime}, A \cup B\right), \forall x \in U$; then,

$$
\begin{aligned}
k_{t}^{\prime}(x) & =\left\{\begin{array}{ll}
0, & t \in A-B, \\
0, & t \in B-A, \\
f_{t}(x) \wedge g_{t}(x), & t \in A \cap B,
\end{array} \quad= \begin{cases}f_{t}(x) \wedge g_{t}(x), & t \in A \cap B, \\
0, & \text { otherwise, }\end{cases} \right. \\
{[(f, A) \tilde{\tilde{n}}(g, B)] \tilde{\tilde{\cap}}(h, C) } & =\left(k^{\prime}, A \cup B\right) \tilde{\tilde{\cap}}(h, C)=\left(r^{\prime}, A \cup B \cup C\right), \\
r_{t}^{\prime}(x) & =\left\{\begin{array}{ll}
k_{t}^{\prime}(x) \wedge h(x), & t \in(A \cap B) \cap C, \\
0, & \text { otherwise, }
\end{array}= \begin{cases}f_{t}(x) \wedge g_{t}(x) \wedge h_{t}(x), & t \in A \cap(B \cap C), \\
0, & \text { otherwise. }\end{cases} \right.
\end{aligned}
$$

Therefore, $r_{t}(x)=r_{t}^{\prime}(x)$, that is, (4) is true.

$$
\begin{aligned}
& (f, A) \tilde{\widetilde{U}}[(g, B) \widetilde{\tilde{n}}(h, C)]=(f, A) \widetilde{\cup}[(\tilde{g}, B \cup C) \tilde{\cap}(\widetilde{h}, B \cup C)] \\
& =(f, A) \tilde{\cup}[(k, B \cup C)]=(s, A \cup B \cup C), \quad \forall a \in A \cup B \cup C, \forall x \in U, \\
& s_{a}(x)=\left\{\begin{array}{ll}
f_{a}(x), & a \in A-(B \cup C), \\
f_{a}(x) \vee k_{a}(x), & a \in A \cap(B \cup C), \\
k_{a}(x), & a \in(B \cup C)-A,
\end{array}= \begin{cases}f_{a}(x), & a \in A-(B \cup C), \\
f_{a}(x) \vee\left(\bar{g}_{a}(x) \wedge \bar{h}_{a}(x)\right), & a \in A \cap(B \cup C), \\
\bar{g}_{a}(x) \wedge \bar{h}_{a}(x), & a \in(B \cup C)-A,\end{cases} \right. \\
& =\left\{\begin{array}{ll}
f_{a}(x), & a \in A-(B \cup C), \\
f_{a}(x) \vee\left(g_{a}(x) \wedge h_{a}(x)\right), & a \in A \cap(B \cap C), \\
f_{a}(x) \vee 0, & a \in A \cap[(B \cup C)-(B \cap C)], \\
g_{a}(x) \wedge h_{a}(x), & a \in(B \cap C)-A, \\
0, & a \in[(B \cup C)-(B \cap C)]-A,
\end{array}= \begin{cases}f_{a}(x), & a \in A-(A \cap B \cap C), \\
f_{a}(x) \vee\left(g_{a}(x) \wedge h_{a}(x)\right), & a \in A \cap B \cap C \\
g_{a}(x) \wedge h_{a}(x), & a \in(B \cap C)-A \\
0, & a \in[(B \cup C)-(B \cap C)]-A,\end{cases} \right. \\
& {[(f, A) \tilde{\widetilde{U}}(g, B)] \tilde{\tilde{n}}[(f, A) \widetilde{\widetilde{U}}(h, C)]=[(f, A) \widetilde{\cup}(g, B)] \tilde{\tilde{n}}[(f, A) \widetilde{\cup}(h, C)]} \\
& =(r, A \cup B) \widetilde{\tilde{n}}(v, A \cup C)=(t, A \cup B \cup C), \quad \forall a \in A \cup B \cup C, \forall x \in U, \\
& t_{a}(x)= \begin{cases}r_{a}(x) \wedge v_{a}(x), & a \in(A \cup B) \cap(A \cup C), \\
0, & a \in(A \cup B \cup C)-(A \cup B) \cap(A \cup C),\end{cases} \\
& = \begin{cases}f_{a}(x), & a \in(A-B) \cap(A-C), \\
f_{a}(x) \wedge\left[f_{a}(x) \vee h_{a}(x)\right], & a \in(A-B) \cap(A \cap C), \\
g_{a}(x) \wedge h_{a}(x), & a \in(B-A) \cap(C-A), \\
{\left[f_{a}(x) \vee g_{a}(x)\right] \wedge f_{a}(x),} & a \in(A \cap B) \cap(A-C), \\
{\left[f_{a}(x) \vee g_{a}(x)\right] \wedge\left[f_{a}(x) \vee h_{a}(x)\right],} & a \in(A \cap B) \cap(A \cap C), \\
0, & a \in(A \cup B \cup C)-(A \cup B) \cap(A \cup C),\end{cases} \\
& = \begin{cases}f_{a}(x), & a \in[(A-B) \cap(A-C)] \cup[(A \cap B) \cap(A-C)] \cup[(A-B) \cap(A \cap C)], \\
f_{a}(x) \vee\left[g_{a}(x) \wedge h_{a}(x)\right], & a \in(A \cap B) \cap(A \cap C), \\
g_{a}(x) \wedge h_{a}(x), & a \in(B-A) \cap(C-A), \\
0, & a \in(A \cup B \cup C)-(A \cup B) \cap(A \cup C) .\end{cases}
\end{aligned}
$$


Therefore, $s_{a}(x)=t_{a}(x)$, that is, (5) is true. The proof of (6) is the same as that of (5).

Example 2. Let the domain be $U=\left\{h^{1}, h^{2}, h^{3}\right\}$, the parameter set be $A=\left\{e_{1}, e_{2}\right\}, B=\left\{e_{2}, e_{3}\right\}$, and $C=\left\{e_{1}, e_{3}\right\} \subset E=\left\{e_{1}\right.$, $\left.e_{2}, e_{3}\right\}$, and the three FSSs on $U$ be

$$
\begin{aligned}
& (f, A)=\left\{e_{1}=\left\{h_{0.1}^{1}, h_{0.3}^{2}, h_{0.5}^{3}\right\}, e_{2}=\left\{h_{0.3}^{1}, h_{0.5}^{2}, h_{0.2}^{3}\right\}\right\}, \\
& (g, B)=\left\{e_{2}=\left\{h_{0.2}^{1}, h_{0.3}^{2}, h_{0.5}^{3}\right\}, e_{3}=\left\{h_{0.3}^{1}, h_{0.4}^{2}, h_{0.6}^{3}\right\}\right\}, \\
& (h, C)=\left\{e_{1}=\left\{h_{0.1}^{1}, h_{0.2}^{2}, h_{0.8}^{3}\right\}, e_{3}=\left\{h_{0.5}^{1}, h_{0.3}^{2}, h_{0.2}^{3}\right\}\right\} .
\end{aligned}
$$

Then,

$$
\begin{aligned}
& (f, A) \tilde{\widetilde{U}}[(g, B) \widetilde{\widetilde{U}}(h, C)]=\left\{e_{1}=\left\{h_{0.1}^{1}, h_{0.3}^{2}, h_{0.8}^{3}\right\}, e_{2}=\left\{h_{0.3}^{1}, h_{0.5}^{2}, h_{0.5}^{3}\right\}, e_{3}=\left\{h_{0.5}^{1}, h_{0.4}^{2}, h_{0.6}^{3}\right\}\right\} \\
& =[(f, A) \tilde{\tilde{U}}(g, B)] \tilde{\tilde{U}}(h, C), \\
& (g, B) \tilde{\tilde{\cap}}(h, C)=\left\{e_{1}=\left\{h_{0}^{1}, h_{0}^{2}, h_{0}^{3}\right\}, e_{2}=\left\{h_{0}^{1}, h_{0}^{2}, h_{0}^{3}\right\}, e_{3}=\left\{h_{0.3}^{1}, h_{0.3}^{2}, h_{0.2}^{3}\right\}\right\}, \\
& (f, A) \widetilde{\tilde{n}}[(g, B) \widetilde{\tilde{n}}(h, C)]=\left\{e_{1}=\left\{h_{0}^{1}, h_{0}^{2}, h_{0}^{3}\right\}, e_{2}=\left\{h_{0}^{1}, h_{0}^{2}, h_{0}^{3}\right\}, e_{3}=\left\{h_{0}^{1}, h_{0}^{2}, h_{0}^{3}\right\}\right\}, \\
& (f, A) \tilde{\tilde{n}}(g, B)=\left\{e_{1}=\left\{h_{0}^{1}, h_{0}^{2}, h_{0}^{3}\right\}, e_{2}=\left\{h_{0.2}^{1}, h_{0.3}^{2}, h_{0.2}^{3}\right\}, e_{3}=\left\{h_{0}^{1}, h_{0}^{2}, h_{0}^{3}\right\}\right\}, \\
& {[(f, A) \tilde{\tilde{n}}(g, B)] \tilde{\tilde{n}}(h, C)=\left\{e_{1}=\left\{h_{0}^{1}, h_{0}^{2}, h_{0}^{3}\right\}, e_{2}=\left\{h_{0}^{1}, h_{0}^{2}, h_{0}^{3}\right\}, e_{3}=\left\{h_{0}^{1}, h_{0}^{2}, h_{0}^{3}\right\}\right\},} \\
& =(f, A) \widetilde{\tilde{n}}[(g, B) \widetilde{\tilde{n}}(h, C)], \\
& (f, A) \tilde{\widetilde{U}}[(g, B) \widetilde{\tilde{n}}(h, C)]=\left\{e_{1}=\left\{h_{0.1}^{1}, h_{0.3}^{2}, h_{0.5}^{3}\right\}, e_{2}=\left\{h_{0.3}^{1}, h_{0.5}^{2}, h_{0.2}^{3}\right\}\right\} \\
& \tilde{\widetilde{U}}\left\{e_{1}=\left\{h_{0}^{1}, h_{0}^{2}, h_{0}^{3}\right\}, e_{2}=\left\{h_{0}^{1}, h_{0}^{2}, h_{0}^{3}\right\}, e_{3}=\left\{h_{0.3}^{1}, h_{0.3}^{2}, h_{0.2}^{3}\right\}\right\} \\
& =\left\{e_{1}=\left\{h_{0.1}^{1}, h_{0.3}^{2}, h_{0.5}^{3}\right\}, e_{2}=\left\{h_{0.3}^{1}, h_{0.5}^{2}, h_{0.2}^{3}\right\}, e_{3}=\left\{h_{0.3}^{1}, h_{0.3}^{2}, h_{0.2}^{3}\right\}\right\}, \\
& {[(f, A) \tilde{\cup}(g, B)]=\left\{e_{1}=\left\{h_{0.1}^{1}, h_{0.3}^{2}, h_{0.5}^{3}\right\}, e_{2}=\left\{h_{0.3}^{1}, h_{0.5}^{2}, h_{0.5}^{3}\right\}, e_{3}=\left\{h_{0.3}^{1}, h_{0.4}^{2}, h_{0.6}^{3}\right\}\right\},} \\
& {[(f, A) \widetilde{\widetilde{U}}(h, C)]=\left\{e_{1}=\left\{h_{0.1}^{1}, h_{0.3}^{2}, h_{0.8}^{3}\right\}, e_{2}=\left\{h_{0.3}^{1}, h_{0.5}^{2}, h_{0.2}^{3}\right\}, e_{3}=\left\{h_{0.5}^{1}, h_{0.3}^{2}, h_{0.2}^{3}\right\}\right\} \text {, }} \\
& {[(f, A) \widetilde{\widetilde{U}}(g, B)] \widetilde{\tilde{n}}[(f, A) \widetilde{\widetilde{U}}(h, C)]} \\
& =\left\{e_{1}=\left\{h_{0.1}^{1}, h_{0.3}^{2}, h_{0.5}^{3}\right\}, e_{2}=\left\{h_{0.3}^{1}, h_{0.5}^{2}, h_{0.2}^{3}\right\}, e_{3}=\left\{h_{0.3}^{1}, h_{0.3}^{2}, h_{0.2}^{3}\right\}\right\} \\
& =(f, A) \widetilde{\tilde{U}}[(g, B) \widetilde{\tilde{n}}(h, C)], \\
& (g, B) \widetilde{\widetilde{U}}(h, C)=\left\{e_{1}=\left\{h_{0.1}^{1}, h_{0.2}^{2}, h_{0.8}^{3}\right\}, e_{2}=\left\{h_{0.2}^{1}, h_{0.3}^{2}, h_{0.5}^{3}\right\}, e_{3}=\left\{h_{0.5}^{1}, h_{0.4}^{2}, h_{0.6}^{3}\right\}\right\}, \\
& (f, A) \tilde{\tilde{n}}[(g, B) \tilde{\tilde{U}}(h, C)]=\left\{e_{1}=\left\{h_{0.1}^{1}, h_{0.2}^{2}, h_{0.5}^{3}\right\}, e_{2}=\left\{h_{0.2}^{1}, h_{0.3}^{2}, h_{0.2}^{3}\right\}, e_{3}=\left\{h_{0}^{1}, h_{0}^{2}, h_{0}^{3}\right\}\right\}, \\
& {[(f, A) \tilde{\tilde{n}}(g, B)] \tilde{\tilde{U}}[(f, A) \widetilde{\tilde{n}}(h, C)]} \\
& =\left\{e_{1}=\left\{h_{0}^{1}, h_{0}^{2}, h_{0}^{3}\right\}, e_{2}=\left\{h_{0.2}^{1}, h_{0.3}^{2}, h_{0.2}^{3}\right\}, e_{3}=\left\{h_{0}^{1}, h_{0}^{2}, h_{0}^{3}\right\}\right\} \\
& \widetilde{\widetilde{U}}\left\{e_{1}=\left\{h_{0.1}^{1}, h_{0.2}^{2}, h_{0.5}^{3}\right\}, e_{2}=\left\{h_{0}^{1}, h_{0}^{2}, h_{0}^{3}\right\}, e_{3}=\left\{h_{0}^{1}, h_{0}^{2}, h_{0}^{3}\right\}\right\} \\
& =\left\{e_{1}=\left\{h_{0.1}^{1}, h_{0.2}^{2}, h_{0.5}^{3}\right\}, e_{2}=\left\{h_{0.2}^{1}, h_{0.3}^{2}, h_{0.2}^{3}\right\}, e_{3}=\left\{h_{0}^{1}, h_{0}^{2}, h_{0}^{3}\right\}\right\} \\
& =(f, A) \widetilde{\widetilde{n}}[(g, B) \widetilde{\widetilde{U}}(h, C)] \text {. }
\end{aligned}
$$

Corollary 1. Let $\left\{\left(g_{k}, B_{k}\right) \mid k \in K\right\}$ be a family of FSSs on Uand $(f, A)$ be a FSS on $U$; then,
(1) $\tilde{\tilde{U}}_{k \in K}\left[(f, A) \tilde{\tilde{ก}}\left(g_{k}, B_{k}\right)\right]=(f, A) \tilde{\tilde{n}}\left[\tilde{\tilde{U}}_{k \in K}\left(g_{k}, B_{k}\right)\right]$

(2) $\tilde{\tilde{ก}}_{k \in K}\left[(f, A) \tilde{\tilde{U}}\left(g_{k}, B_{k}\right)\right]=(f, A) \tilde{\tilde{U}}\left[\tilde{\tilde{n}}_{k \in K}\left(g_{k}, B_{k}\right)\right]$ 
Proof (1)

$$
\begin{aligned}
& \underset{\widetilde{\widetilde{U}}}{\tilde{\mathrm{C}}}\left[(f, A) \tilde{\tilde{\cap}}\left(g_{k}, B_{k}\right)\right]=\underset{k \in K}{\tilde{U}}\left(h_{k}, A \cup B_{k}\right)=\left(r, A \cup \underset{k \in K}{\cup} B_{k}\right), \quad \forall a \in A \cup\left(\underset{k \in K}{\cup} B_{k}\right), \forall x \in U, \\
& r_{a}(x)=\vee_{k \in K} h_{k a}(x)= \begin{cases}\vee_{k \in K}\left(f_{a}(x) \wedge g_{k a}(x)\right), & a \in \underset{k \in K}{\cup}\left(A \cap B_{k}\right), \\
\vee_{k \in K}\left(f_{a}(x) \wedge 0\right), & a \in A \cup \underset{k \in K}{\cup} B_{k}-\underset{k \in K}{\cup}\left(A \cap B_{k}\right),\end{cases} \\
& = \begin{cases}f_{a}(x) \wedge\left(\vee_{k \in K} g_{k a}(x)\right), & a \in \underset{k \in K}{\cup}\left(A \cap B_{k}\right), \\
0, & a \in A \cup \underset{k \in K}{\cup} B_{k}-\cup_{k \in K}\left(A \cap B_{k}\right),\end{cases}
\end{aligned}
$$

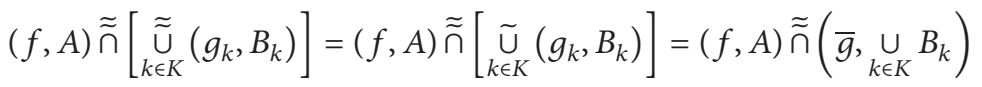

$$
\begin{aligned}
& =\left(s, A \cup\left(\cup_{k \in K} B_{k}\right)\right), \quad \forall a \in A \cup\left(\cup_{k \in K} B_{k}\right), \forall x \in U, \\
& s_{a}(x)= \begin{cases}f_{a}(x) \wedge \bar{g}_{a}(x), & a \in A \cap\left(\cup_{k \in K} B_{k}\right), \\
0, & a \in A \cup\left(\cup_{k \in K} B_{k}\right)-A \cap\left(\cup_{k \in K} B_{k}\right),\end{cases} \\
& = \begin{cases}f_{a}(x) \wedge\left(\vee_{k \in K} g_{k a}(x)\right), & a \in \underset{k \in K}{\cup}\left(A \cap B_{k}\right), \\
0, & a \in A \cup \underset{k \in K}{\cup} B_{k}-\underset{k \in K}{\cup}\left(A \cap B_{k}\right),\end{cases} \\
& =r_{a}(x) .
\end{aligned}
$$

Therefore, (1) is true.

The proof method of (2) is the same as that of (1).

\section{Generalization of Remainder Operation of FSS}

Definition 10. The generalized remainder of a $\operatorname{FSS}(f, A)$ on Uis $\left(f^{C}, E\right), \quad A \subset E$, where $\tilde{f^{c}}: E \longrightarrow F(U) \cdot f_{a}^{\tilde{c}}(x)=$ $\left\{\begin{array}{ll}1-f_{a}(x), & a \in A, \\ 1, & a \notin A,\end{array} \forall x \in U\right.$, denoted by $\left(f^{\widetilde{C}}, E\right)=(f, A)^{\widetilde{C}}$.
Example 3. Let the domain be $U=\left\{h^{1}, h^{2}, h^{3}\right\}$, the parameter set be $A=\left\{e_{1}, e_{2}\right\}$ and $B=\left\{e_{2}, e_{3}\right\} \subset E=\left\{e_{1}, e_{2}, e_{3}\right\}$, and the two FSSs on $U$ be

$$
\begin{aligned}
(h, C) & =\left\{e_{1}=\left\{h_{0.2}^{1}, h_{0.5}^{2}, h_{0.7}^{3}\right\}, e_{2}=\left\{h_{0.4}^{1}, h_{0.8}^{2}, h_{0.2}^{3}\right\}\right\}, \\
(k, D) & =\left\{e_{2}=\left\{h_{0.3}^{1}, h_{0.4}^{2}, h_{0.6}^{3}\right\}, e_{3}=\left\{h_{0.1}^{1}, h_{0.2}^{2}, h_{0.3}^{3}\right\}\right\}, \\
(h, C)^{\tilde{C}} & =\left\{e_{1}=\left\{h_{0.8}^{1}, h_{0.5}^{2}, h_{0.3}^{3}\right\}, e_{2}=\left\{h_{0.6}^{1}, h_{0.2}^{2}, h_{0.8}^{3}\right\}, e_{3}=\left\{h_{1}^{1}, h_{1}^{2}, h_{1}^{3}\right\}\right\}, \\
(k, D)^{\widetilde{C}} & =\left\{e_{1}=\left\{h_{1}^{1}, h_{1}^{2}, h_{1}^{3}\right\}, e_{2}=\left\{h_{0.7}^{1}, h_{0.6}^{2}, h_{0.4}^{3}\right\}, e_{3}=\left\{h_{0.9}^{1}, h_{0.8}^{2}, h_{0.7}^{3}\right\}\right\} .
\end{aligned}
$$

Theorem 2. The generalization of the union and intersection operations of fuzzy FSSs satisfies De Morgan's law, that is, let $(h, A),(k, B)$ be FSSs on $U$; then, there are

(1) $[(h, A) \widetilde{\widetilde{U}}(k, B)]^{\widetilde{C}}=(h, A)^{\tilde{C}} \widetilde{\cap}(k, B)^{\tilde{C}}$
(2) $[(h, A) \widetilde{\widetilde{\cap}}(k, B)]^{\widetilde{C}}=(h, A)^{\widetilde{C}} \widetilde{\widetilde{U}}(k, B)^{\widetilde{C}}$

Proof. $\widetilde{C}^{(1)} \quad[(h, A) \widetilde{\widetilde{U}}(k, B)]^{\widetilde{C}}=[(h, A) \widetilde{U}(k, B)]^{\widetilde{C}}=[(r$, $A \cup B)]^{C}$. 
From $\forall x \in U$,

$$
\begin{aligned}
& r_{a}(x)=\left\{\begin{array}{ll}
h_{a}(x), & a \in A-B, \\
k_{a}(x)
\end{array}, a \in B-A, h_{a}(x) \vee k_{a}(x), a \notin A \cup B,\right. \\
& r_{a}^{c}(x)=\left\{\begin{array}{ll}
1-r_{a}(x), a \in A \cup B \\
1, a \notin A \cup B
\end{array}= \begin{cases}1-\left(h_{a}(x) \vee k_{a}(x)\right), & a \in A \cap B, \\
\left(1-h_{a}(x)\right) \wedge 1, & a \in A-B, \\
1 \wedge\left(1-k_{a}(x)\right), & a \in B-A, \\
1 \wedge 1, & a \notin A \cup B,\end{cases} \right. \\
& \tilde{h_{a}^{c}}(x)= \begin{cases}1-h_{a}(x), & a \in A, \\
1, & a \notin A,\end{cases} \\
& \tilde{k_{a}^{c}}(x)= \begin{cases}1-k_{a}(x), & a \in B, \\
1, & a \notin B,\end{cases} \\
& (h, A)^{\tilde{C} \widetilde{\widetilde{ }}}(k, B)^{\widetilde{C}}=(s, E)^{\tilde{C}}, \\
& \tilde{s}_{a}^{c}(x)= \begin{cases}\left(1-h_{a}(x)\right) \wedge\left(1-k_{a}(x)\right), & a \in A \cap B, \\
\left(1-h_{a}(x)\right) \wedge 1, & a \in A-B, \\
1 \wedge\left(1-k_{a}(x)\right), & a \in B-A, \\
1 \wedge 1, & a \notin A \cup B,\end{cases} \\
& \left(1-h_{a}(x)\right) \wedge\left(1-k_{a}(x)\right)=\min \left\{1-h_{a}(x), 1-k_{a}(x)\right\}=1-\max \left\{h_{a}(x), k_{a}(x)\right\}=1-\left(h_{a}(x) \vee k_{a}(x)\right)
\end{aligned}
$$

we get $\tilde{r}_{\underline{a}}^{\tilde{c}}(x)=\tilde{s}_{a}^{\tilde{c}}(x)$ and $[(h, A) \widetilde{\widetilde{U}}(k, B)]^{\widetilde{C}}=(h$,

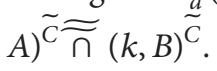

The proof of (2) is similar to that of (1).
Example 4. Let the domain be $U=\left\{h^{1}, h^{2}, h^{3}\right\}$, the parameter set be $A=\left\{e_{1}, e_{2}\right\}$ and $B=\left\{e_{2}, e_{3}\right\} \subset E=\left\{e_{1}, e_{2}, e_{3}\right\}$, and the two FSSs on $U$ be

$$
\begin{aligned}
& (h, C)=\left\{e_{1}=\left\{h_{0.2}^{1}, h_{0.5}^{2}, h_{0.7}^{3}\right\}, e_{2}=\left\{h_{0.4}^{1}, h_{0.8}^{2}, h_{0.2}^{3}\right\}\right\}, \\
& (k, D)=\left\{e_{2}=\left\{h_{0.3}^{1}, h_{0.4}^{2}, h_{0.6}^{3}\right\}, e_{3}=\left\{h_{0.1}^{1}, h_{0.2}^{2}, h_{0.3}^{3}\right\}\right\}, \\
& (h, C) \widetilde{\widetilde{U}}(k, D)=\left\{e_{1}=\left\{h_{0.2}^{1}, h_{0.5}^{2}, h_{0.7}^{3}\right\}, e_{2}=\left\{h_{0.4}^{1}, h_{0.8}^{2}, h_{0.6}^{3}\right\}, e_{3}=\left\{h_{0.1}^{1}, h_{0.2}^{2}, h_{0.3}^{3}\right\}\right\}, \\
& (h, C) \widetilde{\widetilde{\cap}}(k, D)=\left\{e_{1}=\left\{h_{0 .}^{1}, h_{0}^{2}, h_{0}^{3}\right\}, e_{2}=\left\{h_{0.3}^{1}, h_{0.4}^{2}, h_{0.2}^{3}\right\}, e_{3}=\left\{h_{0}^{1}, h_{0}^{2}, h_{0}^{3}\right\}\right\}, \\
& {[(h, C) \widetilde{\widetilde{U}}(k, D)]^{C}=\left\{e_{1}=\left\{h_{0.8}^{1}, h_{0.5}^{2}, h_{0.3}^{3}\right\}, e_{2}=\left\{h_{0.6}^{1}, h_{0.2}^{2}, h_{0.4}^{3}\right\}, e_{3}=\left\{h_{0.9}^{1}, h_{0.8}^{2}, h_{0.7}^{3}\right\}\right\},} \\
& {[(h, C) \widetilde{\widetilde{\cap}}(k, D)]^{C}=\left\{e_{1}=\left\{h_{1}^{1}, h_{1}^{2}, h_{1}^{3}\right\}, e_{2}=\left\{h_{0.7}^{1}, h_{0.6}^{2}, h_{0.8}^{3}\right\}, e_{3}=\left\{h_{1}^{1}, h_{1}^{2}, h_{1}^{3}\right\}\right\},} \\
& (h, C)^{C}=\left\{e_{1}=\left\{h_{0.8}^{1}, h_{0.5}^{2}, h_{0.3}^{3}\right\}, e_{2}=\left\{h_{0.6}^{1}, h_{0.2}^{2}, h_{0.8}^{3}\right\}, e_{3}=\left\{h_{1}^{1}, h_{1}^{2}, h_{1}^{3}\right\}\right\}, \\
& (k, D)^{C}=\left\{e_{1}=\left\{h_{1}^{1}, h_{1}^{2}, h_{1}^{3}\right\}, e_{2}=\left\{h_{0.7}^{1}, h_{0.6}^{2}, h_{0.4}^{3}\right\}, e_{3}=\left\{h_{0.9}^{1}, h_{0.8}^{2}, h_{0.7}^{3}\right\}\right\}, \\
& {[(h, C)]^{C} \widetilde{\widetilde{\cap}}[(k, D)]^{C}=\left\{e_{1}=\left\{h_{0.8}^{1}, h_{0.5}^{2}, h_{0.3}^{3}\right\}, e_{2}=\left\{h_{0.6}^{1}, h_{0.2}^{2}, h_{0.4}^{3}\right\}, e_{3}=\left\{h_{0.9}^{1}, h_{0.8}^{2}, h_{0.7}^{3}\right\}\right\}} \\
& =[(h, C) \widetilde{\widetilde{U}}(k, D)]^{C} \text {, } \\
& {[(h, C)]^{C} \widetilde{\widetilde{U}}[(k, D)]^{C}=\left\{e_{1}=\left\{h_{1}^{1}, h_{1}^{2}, h_{1}^{3}\right\}, e_{2}=\left\{h_{0.7}^{1}, h_{0.6}^{2}, h_{0.8}^{3}\right\}, e_{3}=\left\{h_{1}^{1}, h_{1}^{2}, h_{1}^{3}\right\}\right\}} \\
& =[(h, C) \widetilde{\widetilde{\cap}}(k, D)]^{C} \text {. }
\end{aligned}
$$




\section{Topology Construction Based on FSS Generalization under Intersection and Union Operation}

Definition 11 (see [13]). Let $F(U ; E)$ be the whole family of fuzzy FSSs with parameter set $E$ on $U ;(\gamma, X) \in F(U ; E)$ and $P(\gamma, X)$ are the whole fuzzy soft subset of $(\gamma, X) . \widetilde{\tau}$ is a subfamily of $P(\gamma, X)$; then, $\widetilde{\tau}$ is a fuzzy soft topology of $(\gamma, X)$. If (1) $\widetilde{\Phi}_{x},(\gamma, X) \in \widetilde{\tau},(2)(f, A),(g, B) \in \widetilde{\tau} \Rightarrow(f, A)$ $\tilde{\cap}(g, B) \in \widetilde{\tau}$, and $(3)\left\{\left(f^{k}, A_{k}\right) \mid k \in K\right\} \subset \widetilde{\tau} \Rightarrow \widetilde{U}_{k \in K}\left(f^{k}\right.$, $\left.A_{k}\right) \in \tilde{\tau}$, then $\left(X_{\gamma}, \widetilde{\tau}\right)$ is a fuzzy soft topology space.

Definition 12 (see [13]). Let $\left(X_{\gamma}, \widetilde{\tau}\right)$ be a fuzzy soft topological space, and the topological space consisting of $(f$, $A) \in P(\gamma, X), \widetilde{\tau}_{(f, A)}=\{(f, A) \widetilde{\cap}(g, B) \mid(g, B) \in \widetilde{\tau}\}$ is called the fuzzy soft topological subspace of $\left(X_{\gamma}, \tilde{\tau}\right)$, denoted as $\left(A_{f}, \widetilde{\tau}_{(f, A)}\right)$.

Definition 13. Let $F(U ; E)$ be the whole family of FSSs with parameter set $E$ on $U ;(\gamma, X) \in F(U ; E)$ and $P(\gamma, X)$ are the whole FSS of $(\gamma, X)$. $\widetilde{\widetilde{\tau}}$ is a subfamily of $P(\gamma, X)$; then, $\widetilde{\widetilde{\tau}}$ is a fuzzy soft topology of $(\gamma, X)$. If $(1) \widetilde{\Phi}_{x},(\gamma, X) \in \widetilde{\widetilde{\tau}},(2)(f, A)$, $(g, B) \in \underset{\widetilde{\tau}}{\widetilde{\tau}} \Rightarrow(f, A) \widetilde{\widetilde{\pi}}(g, B) \in \widetilde{\tilde{\tau}}$, and $(3)\left\{\left(f_{k}, A_{k}\right) \mid k \in K\right\}$ $\subset \widetilde{\widetilde{\tau}} \Rightarrow \widetilde{\widetilde{U}}_{k \in K}\left(f_{k}, A_{k}\right) \in \widetilde{\widetilde{\tau}}$, then $\left(X_{\gamma}, \widetilde{\tilde{\tau}}\right)$ is a fuzzy soft topology space.

Theorem 3. Let $F(U ; E)$ be the whole family of fuzzy soft sets with parameter set $E$ on $U ;(\gamma, X) \in F(U ; E)$ and $P(\gamma, X)$ are the whole FSS of $(\gamma, X)$.

$\tilde{\tau}$ is a subfamily of $P(\gamma, X)$, if

(1) $\widetilde{\Phi}_{X},(\gamma, X) \in \widetilde{\tau}$
(2) $(f, A),(g, B) \in \widetilde{\tau} \Rightarrow(f, A) \widetilde{\cap}(g, B) \in \widetilde{\tau}$

(3) $\left\{\left(f_{k}, A_{k}\right) \mid k \in K\right\} \subset \widetilde{\tau} \Rightarrow \widetilde{U}_{k \in K}\left(f_{k}, A_{k}\right) \in \tilde{\tau}$

(4) $\forall(f, A),(g, B) \in \widetilde{\tau}, \quad \widetilde{\Phi}_{(A \cup B)-(A \cap B)} \in \widetilde{\tau}$, then $\tilde{\tau}$ constitute the fuzzy soft topology under Definition 13

Remark 4

(1) $(f, A) \widetilde{\widetilde{\cap}}(g, B)=[(f, A) \widetilde{\cap}(g, B)] \widetilde{\cup} \widetilde{\Phi}_{(A \cup B)-(A \cap B)}$, $\widetilde{\widetilde{U}}_{k \in K}\left(f_{k}, A_{k}\right)=\widetilde{U}_{k \in K}\left(f_{k}, A_{k}\right)$, Therefore, it can be seen that Theorem 2 holds.

(2) Comparing Definition 10 with Definition 11, it can be seen that the conditions required for topology structure construction based on intersection and union operation in the traditional sense of FSS are relatively weaker than that based on the generalized intersection and union operation of FSS, but the intersection and union operation in the traditional sense of FSS does not satisfy De Morgan's law. This makes it very difficult to further study the structure and properties of fuzzy soft topology. The intersection and union operations in the generalized sense of fuzzy FSS satisfy De Morgan's law, which will bring great convenience for further study the structure of fuzzy soft topology.

Example 5. Let $U=\left\{h^{1}, h^{2}, h^{3}, h^{4}, h^{5}\right\}$ be the domain and $E=\left\{e_{1}, e_{2}, e_{3}\right\}$ be the set of parameters, and the fuzzy soft set is

$$
\begin{aligned}
(\gamma, X) & =\left\{e_{1}=\left\{h_{0.2}^{1}, h_{1}^{2}, h_{0.1}^{3}, h_{1}^{4}, h_{1}^{5}\right\}, e_{2}=\left\{h_{0}^{1}, h_{0.3}^{2}, h_{0.6}^{3}, h_{0.6}^{4}, h_{0.4}^{5}\right\}, e_{3}=\left\{h_{0.2}^{1}, h_{0.3}^{2}, h_{0.8}^{3}, h_{1}^{4}, h_{0.6}^{5}\right\}\right\}, \\
\widetilde{\tau} & =\left\{\widetilde{\Phi}_{X},(\gamma, X),\left\{e_{1}=\left\{h_{0.2}^{1}, h_{1}^{2}, h_{0.1}^{3}, h_{0.2}^{4}, h_{0.5}^{5}\right\}, e_{2}=\left\{h_{0}^{1}, h_{0.3}^{2}, h_{0.6}^{3}, h_{0.2}^{4}, h_{0.3}^{5}\right\}, e_{3}=\left\{h_{0}^{1}, h_{0}^{2}, h_{0}^{3}, h_{0}^{4}, h_{0}^{5}\right\}\right\},\right. \\
\left\{e_{3}\right. & \left.=\left\{h_{0.2}^{1}, h_{0.3}^{2}, h_{0.8}^{3}, h_{0}^{4}, h_{0}^{5}\right\}\right\},\left\{e_{3}=\left\{h_{0}^{1}, h_{0}^{2}, h_{0}^{3}, h_{0}^{4}, h_{0}^{5}\right\}\right\}, \\
\left\{e_{1}\right. & =\left\{h_{0}^{1}, h_{0}^{2}, h_{0}^{3}, h_{0}^{4}, h_{0}^{5}\right\},\left\{e_{2}=\left\{h_{0}^{1}, h_{0}^{2}, h_{0}^{3}, h_{0}^{4}, h_{0}^{5}\right\}\right\}, \\
\left\{e_{1}\right. & =\left\{h_{0}^{1}, h_{0}^{2}, h_{0}^{3}, h_{0}^{4}, h_{0}^{5}\right\},\left\{e_{2}=\left\{h_{0}^{1}, h_{0}^{2}, h_{0}^{3}, h_{0}^{4}, h_{0}^{5}\right\}, e_{3}=\left\{h_{0.2}^{1}, h_{0.3}^{2}, h_{0.8}^{3}, h_{0}^{4}, h_{0}^{5}\right\}\right\} .
\end{aligned}
$$

It is easy to verify that $\tilde{\widetilde{\tau}}$ satisfies all the conditions in Definition 4.3 and constitutes a fuzzy soft topology under Definition 4.3.

Definition 14 If $(f, A) \widetilde{c}(\gamma, X)$ is a FSS, for $\forall x \in U, \forall a$ $\in A$, and $f_{a}(x)=\gamma_{a}(x)$, then $(f, A)$ is the limit of $(\gamma, X)$ on $A$, denoted as $(f, A)=\left(\left.\gamma\right|_{A}, A\right)$.

Theorem 4. Let $(f, A) \in P(\gamma, X), \widetilde{\Phi}_{A} \in \tilde{\widetilde{\tau}}$, and $\left(\left.\gamma\right|_{A}, A\right) \in \widetilde{\widetilde{\tau}}$, where $\left(X_{\gamma}, \tilde{\widetilde{\tau}}\right)$ is the fuzzy soft topological space under Definition 10, and then, $\widetilde{\widetilde{\tau}}_{(f, A)}=\{(f, A) \widetilde{\widetilde{\Pi}}(g, B) \mid(g, B) \in \widetilde{\tilde{\tau}}\}$ forms the fuzzy soft topology of $(f, A)$ under Definition 10.
Proof

(1) From $\Phi_{A} \in \tilde{\widetilde{\tau}}$ and $\widetilde{\Phi}_{A}=(f, A) \widetilde{\widetilde{n}} \widetilde{\Phi}_{A}$, we get $\widetilde{\Phi}_{A} \epsilon$ $\tilde{\widetilde{\tau}}_{(f, A)}$; from $\left(\left.\gamma\right|_{A}, A\right) \in \tilde{\widetilde{\tau}},(f, A)=(f, A) \widetilde{\widetilde{\Pi}}\left(\left.\gamma\right|_{A}, A\right)$, we get $(f, A) \in \widetilde{\tilde{\tau}}_{(f, A)}$.

(2) Let $\left(h_{1}, C_{1}\right),\left(h_{2}, C_{2}\right) \in \tilde{\widetilde{\tau}}_{(f, A)}$; then, $\left(g_{1}, B_{1}\right),\left(g_{2}\right.$, $\left.B_{2}\right) \in \tilde{\widetilde{\tau}}$, such that $\left(h_{1}, C_{1}\right)=(f, A) \widetilde{\widetilde{n}}\left(g_{1}, B_{1}\right),\left(h_{2}\right.$, $\left.C_{2}\right)=(f, A) \widetilde{\widetilde{n}}\left(g_{2}, B_{2}\right)$, and $\left(h_{1}, C_{1}\right) \widetilde{\widetilde{n}}\left(h_{2}, C_{2}\right)=$ $\left[(f, A) \widetilde{\widetilde{\Pi}}\left(g_{1}, B_{1}\right)\right] \widetilde{\widetilde{n}}\left[(f, A) \widetilde{\widetilde{\pi}}\left(g_{2}, B_{2}\right)\right]=(f, A)$ $\widetilde{\widetilde{n}}\left[\left(g_{1}, B_{1}\right) \widetilde{\widetilde{n}}\left(g_{2}, B_{2}\right)\right]$; from $\left(g_{1}, B_{1}\right)$ 吕 $\left(g_{2}, B_{2}\right) \epsilon$ $\tilde{\widetilde{\tau}}$, we get $\left(h_{1}, C_{1}\right) \widetilde{\widetilde{\cap}}\left(h_{2}, C_{2}\right) \in \widetilde{\widetilde{\tau}}_{(f, A)}$. 
(3) Let $\left\{\left(h_{k}, C_{k}\right) \mid k \in K\right\}$ be a subfamily of $\widetilde{\widetilde{\tau}}_{(f, A)}$, and for $\forall k \in K$, there is $\left(g_{k}, B_{k}\right) \in \widetilde{\widetilde{\tau}}$, and we make $\left(h_{k}, C_{k}\right)=$ $(f, A) \widetilde{\widetilde{\cap}}\left(g_{k}, B_{k}\right)$, and then, we get $\tilde{\tilde{U}}_{k \in K}\left(h_{k}, C_{k}\right)=$ $\widetilde{\tilde{U}}_{k \in K}\left[(f, A) \widetilde{\tilde{\cap}}\left(g_{k}, B_{k}\right)\right]=(f, A) \widetilde{\widetilde{\cap}}\left[\tilde{\widetilde{U}}_{k \in K}\left(g_{k}, B_{k}\right)\right]$. $\widetilde{\widetilde{\tau}}_{(f, A)}$.

Because $\widetilde{\widetilde{U}}_{k \in K}\left(g_{k}, B_{k}\right) \in \tilde{\widetilde{\tau}}$, we get $\widetilde{\widetilde{U}}_{k \in K}\left(h_{k}, C_{k}\right) \epsilon$

Therefore, $\widetilde{\widetilde{\tau}}_{(f, A)}$ is the fuzzy soft topology of fuzzy soft subset $(f, A)$ under Definition 10 .

\section{Topology Construction Based on Weak and Strong Fuzzy Soft Sets}

In the analysis and research of FSS operation, F.B. Hua and Y.P. Wang improved the intersection and union operation of FSS and gave the concepts of weak FSS and strong FSS based on the different parameter sets of fuzzy FSS. At the same time, the intersection and union operations of FSSs are improved and the properties of the operations are given. It is concluded that both weak FSSs and strong FSSs satisfy De Morgan's law under the improved intersection and union operations.

Definition 15 (see [20]). Set $U$ as the domain, $A$ as the parameter set, $A \subset E, F(U)$ as the whole FSS on $U$, and $(f, A)$ as the FSS $f_{a}(x), \quad$ Let $_{A}, f: E \longrightarrow F(U)$, $\forall x \in U, \forall a \in E, f_{a}(x)=\left\{\begin{array}{ll}f_{a}(x), & a \in A, \\ 0, & a \notin A .\end{array}\right.$. Then, let $(f, A)$

Remark 5. If the weak FSS is based on parameter $a \in E, a \notin A$, the FSS $(f, A)$ is considered to have no influence on the decision.

Remark 6. $(\breve{f}, A)$ is a weak FSS on $U$, i.e., $(\breve{f}, A)=(f, A)$ $\cup \widetilde{\Phi}_{E-A}$.

Definition 16 (see [20]). Set $U$ as the domain, $A$ as the parameter set, $A \subset E, F(U)$ as the whole FSS on $U$, and $(f$, $A)$ as the FSS on $U$. Let $f: E \longrightarrow F(U), \forall x \in U, \forall a \in E$, $\hat{f}_{a}(x)=\left\{\begin{array}{ll}f_{a}(x), & a \in A, \\ 1, & a \notin A .\end{array}\right.$ Then, let $(\hat{f}, A)$ be a strong FSS on $U$.

Remark 7. If the strong FSS is based on parameter $a \in E$ and $a \notin A$, the FSS $(f, A)$ is considered to have an absolute influence on decision-making.
Remark 8. $(\hat{f}, A)$ is a strong FSS on $U$, i.e., $(\tilde{f}, A)=(f$, A) $\cup \widetilde{U}_{E-A}$.

Definition 17 (see [20]). For two weakly FSSs $(\hat{f}, A)$ and $(\widehat{g}, B)$ on domain $U$, the union and intersection of them are defined as follows:

(1) The union of $(\breve{f}, A)$ and $(\breve{g}, B)$ is defined as a weakly FSS $(h, C)$ on $U$, denoted as $(h, C)=(f, A) \cup_{W}(\breve{g}$, $B$ ), where $C=A \cup B, \forall x \in U, \forall a \in E$, and $h_{a}(x)=$ $f_{a}(x) \vee \breve{g}_{a}(x)$.

(2) The intersection of $(\breve{f}, A)$ and $(\breve{g}, B)$ is defined as a weakly FSS $(\breve{h}, C)$ on $U$, denoted as $(\breve{h}, C)=(\breve{f}, A)$ $\cap_{W}(\breve{g}, B)$, where $C=A \cap B, \forall x \in U, \forall a \in E$, and $\breve{h}_{a}$ $(x)=\breve{f}_{a}(x) \wedge \breve{g}_{a}(x)$.

Remark 9. In the definition of the union and intersection operation of weak FSSs, the operation of parameter sets is consistent with that of the traditional FSSs.

Definition 18 (see [20]). For two strong FSSs $(\breve{f}, A)$ and $(\mathcal{g}, B)$ on domain $U$, the union and intersection of them are defined as follows.

(3) The union of $(\hat{f}, A)$ and $(\hat{g}, B)$ is defined as a strong FSS $(\hat{h}, C)$ on $U$, denoted as $(\widehat{h}, C)=(\widehat{f}, A) \cup_{S}$ $(\widehat{g}, B)$, where $C=A \cap B \neq \Phi, \forall x \in U, \forall a \in E$, and $\widehat{h}_{a}$ $(x)=\hat{f}_{a}(x) \vee \hat{g}_{a}(x)$.

(4) The intersection of $(\hat{f}, A)$ and $(\hat{g}, B)$ is defined as a strongly FSS $(\widehat{h}, C)$ on $U$, denoted as $(\widehat{h}, C)=(\widehat{f}$, A) $\cap_{S}(\widehat{g}, B)$, where $C=A \cup B, \forall x \in U, \forall a \in E$, and $h_{a}(x)=f_{a}(x) \wedge \widehat{g}_{a}(x)$.

Remark 10. In the definition of the union and intersection operation of strong FSS, the operation of parameter set and the traditional union and intersection operation of FSS have changed.

Example 6. Let the domain be $U=\left\{h^{1}, h^{2}, h^{3}\right\}$, the parameter set be $A=\left\{e_{1}, e_{2}\right\}$ and $B=\left\{e_{2}, e_{3}\right\} \subset E=\left\{e_{1}, e_{2}, e_{3}\right\}$, and the two FSSs on $U$ be

$$
\begin{aligned}
& (f, A)=\left\{e_{1}=\left\{h_{0.5}^{1}, h_{0.3}^{2}, h_{0.7}^{3}\right\}, e_{2}=\left\{h_{0.6}^{1}, h_{0.3}^{2}, h_{0.2}^{3}\right\}\right\}, \\
& (g, B)=\left\{e_{2}=\left\{h_{0.3}^{1}, h_{0.4}^{2}, h_{0.8}^{3}\right\}, e_{3}=\left\{h_{0.1}^{1}, h_{0.3}^{2}, h_{0.5}^{3}\right\}\right\}, \\
& (\widetilde{f}, A)=(f, A) \cup \widetilde{\Phi}_{E-A}=\left\{e_{1}=\left\{h_{0.5}^{1}, h_{0.3}^{2}, h_{0.7}^{3}\right\}, e_{2}=\left\{h_{0.6}^{1}, h_{0.3}^{2}, h_{0.2}^{3}\right\}, e_{3}=\left\{h_{0}^{1}, h_{0}^{2}, h_{0}^{3}\right\}\right\}, \\
& (\widetilde{g}, B)=(g, B) \cup \widetilde{U}_{E-B}=\left\{e_{1}=\left\{h_{1}^{1}, h_{1}^{2}, h_{1}^{3}\right\}, e_{2}=\left\{h_{0.3}^{1}, h_{0.4}^{2}, h_{0.8}^{3}\right\}, e_{3}=\left\{h_{0.1}^{1}, h 2_{0.3}^{2}, h_{0.5}^{3}\right\}\right\},
\end{aligned}
$$

respectively. 
Definition 19. Let $F(U ; E)$ be the whole family of FSSs with $E$ as the parameter set and $U$ as the domain. The weak FSS $(\breve{\gamma}, X) \in F(U ; E)$ and $P(\widetilde{\gamma}, X)$ is the whole weak fuzzy soft subset of $(\widetilde{\gamma}, X) . \widetilde{\tau}$ is a subfamily of $P(\widetilde{\gamma}, X)$; then, $\widetilde{\tau}$ is a weak fuzzy soft topology of $(\widetilde{\gamma}, X)$ if

(1) $\widetilde{\Phi}_{X},(\breve{\gamma}, X) \in \breve{\tau}$

(2) $(\breve{f}, A),(\breve{g}, B) \in \breve{\tau} \Longrightarrow(\breve{f}, A) \cap_{W}(\breve{g}, B) \in \widetilde{\tau}$

(3) $\left\{\left(\breve{f}_{k}, A_{k}\right) \mid k \in K\right\} \subset \breve{\tau} \Longrightarrow \cup_{W_{k \in K}}\left(\breve{f}_{k}, A_{k}\right) \in \check{\tau}$

$(X, \widetilde{\tau})$ is called a weakly fuzzy soft topology space.

Definition 20. Let $F(U ; E)$ be the whole family of FSSs with $E$ as the parameter set and $U$ as the domain. The strong FSS $(\widehat{\gamma}, X) \in F(U ; E)$ and $P(\bar{\gamma}, X)$ is the whole strong fuzzy soft subset of $(\widehat{\gamma}, X) . \bar{\tau}$ is a subfamily of $P(\widehat{\gamma}, X)$; then, $\bar{\tau}$ is a strong fuzzy soft topology of $(\bar{\gamma}, X)$ if

(1) $\widetilde{\Phi}_{X},(\bar{\gamma}, X) \in \bar{\tau}$

(2) $(f, A),(g, B) \in \bar{\tau} \Rightarrow(f, A) \cap_{S}(g, B) \in \widehat{\tau}$

(3) $\left\{\left(f_{k}, A_{k}\right) \mid k \in K\right\} \subset \bar{\tau} \Rightarrow \cup_{S_{k \in K}}\left(f_{k}, A_{k}\right) \in \widehat{\tau}$

$(X, \widehat{\tau})$ is called a strong fuzzy soft topology space.

Example 7. Let the domain be $U=\left\{h_{1}, h_{2}\right\}$ and the parameter set be $E=\left\{e_{1}, e_{2}, e_{3}\right\}$ and $(\gamma, X)=\left\{e_{1}=\left\{h_{0.2}^{1}, h_{0.5}^{2}\right\}\right.$, $\left.e_{2}=\left\{h_{0.3}^{1}, h_{0.4}^{2}\right\}\right\} ; \quad$ then, $\quad(\breve{\gamma}, X)=\left\{e_{1}=\left\{h_{0.2}^{1}, h_{0.5}^{2}\right\}, e_{2}=\right.$ $\left.\left\{h_{0.3}^{1}, h_{0.4}^{2}\right\}, e_{3}=\left\{h_{0}^{1}, h_{0}^{2}\right\}\right\}$.

Set

$$
\begin{aligned}
\breve{\tau}= & \left\{(\widetilde{\gamma}, X), \widetilde{\Phi}_{X},\left\{e_{1}=\left\{h_{0.2}^{1}, h_{0.3}^{2}\right\}, e_{2}=\left\{h_{0.2}^{1}, h_{0.4}^{2}\right\}, e_{3}=\left\{h_{0}^{1}, h_{0}^{2}\right\}\right\},\left\{e_{1}=\left\{h_{0.1}^{1}, h_{0.2}^{2}\right\}, e_{2}=\left\{h_{0.2}^{1}, h_{0.3}^{2}\right\}, e_{3}=\left\{h_{0}^{1}, h_{0}^{2}\right\}\right\},\right. \\
& \left.\cdot\left\{e_{1}=\left\{h_{0.2}^{1}, h_{0.2}^{2}\right\}, e_{2}=\left\{h_{0.2}^{1}, h_{0.3}^{2}\right\}, e_{3}=\left\{h_{0}^{1}, h_{0}^{2}\right\}\right\}\right\}
\end{aligned}
$$

and directly verify by Definition 19 , and we can know that $\breve{\tau}$ is the weak fuzzy soft topology of $(\bar{\gamma}, X)$. It is worth noting that

$$
\begin{aligned}
\tilde{\tau}= & \left\{(\gamma, X), \widetilde{\Phi}_{X},\left\{e_{1}=\left\{h_{0.2}^{1}, h_{0.3}^{2}\right\}, e_{2}=\left\{h_{0.2}^{1}, h_{0.4}^{2}\right\}\right\},\left\{e_{1}=\left\{h_{0.1}^{1}, h_{0.2}^{2}\right\}, e_{2}=\left\{h_{0.2}^{1}, h_{0.3}^{2}\right\}\right\},\right. \\
& \left.\cdot\left\{e_{1}=\left\{h_{0.2}^{1}, h_{0.2}^{2}\right\}, e_{2}=\left\{h_{0.2}^{1}, h_{0.3}^{2}\right\}\right\}\right\}
\end{aligned}
$$

is the fuzzy soft topology of $(\gamma, X)$.

Example 8. Let the domain be $U=\left\{h_{1}, h_{2}\right\}$ and the parameter set be $E=\left\{e_{1}, e_{2}, e_{3}\right\}$ and $(\gamma, X)=\left\{e_{1}=\left\{h_{0.2}^{1}, h_{0.5}^{2}\right\}\right.$, $\left.e_{2}=\left\{h_{0.3}^{1}, h_{0.4}^{2}\right\}\right\}$; then, $(\bar{\gamma}, X)=\left\{e_{1}=\left\{h_{0.2}^{1}, h_{0.5}^{2}\right\}, e_{2}=\left\{h_{0.3}^{1}\right.\right.$, $\left.h_{0.4}^{2}\right\}, e_{3}=\left\{h_{1}^{1}, h_{1}^{2}\right\}$. Set

$$
\begin{aligned}
\widehat{\tau}= & \left\{(\bar{\gamma}, X), \widetilde{\Phi}_{X},\left\{e_{1}=\left\{h_{0.2}^{1}, h_{0.3}^{2}\right\}, e_{2}=\left\{h_{0.2}^{1}, h_{0.4}^{2}\right\}, e_{3}=\left\{h_{1}^{1}, h_{1}^{2}\right\}\right\},\left\{e_{1}=\left\{h_{0.1}^{1}, h_{0.2}^{2}\right\}, e_{2}=\left\{h_{0.2}^{1}, h_{0.3}^{2}\right\}, e_{3}=\left\{h_{1}^{1}, h_{1}^{2}\right\}\right\}\right. \\
& \left.\cdot\left\{e_{1}=\left\{h_{0.2}^{1}, h_{0.2}^{2}\right\}, e_{2}=\left\{h_{0.2}^{1}, h_{0.3}^{2}\right\}, e_{3}=\left\{h_{1}^{1}, h_{1}^{2}\right\}\right\}\right\}
\end{aligned}
$$

and directly verify by Definition 20, and we can know that $\bar{\tau}$ is the strong fuzzy soft topology of $(\hat{\gamma}, X)$. It is worth noting that

$$
\begin{aligned}
\tilde{\tau}= & \left\{(\gamma, X), \widetilde{\Phi}_{X},\left\{e_{1}=\left\{h_{0.2}^{1}, h_{0.3}^{2}\right\}, e_{2}=\left\{h_{0.2}^{1}, h_{0.4}^{2}\right\}\right\},\left\{e_{1}=\left\{h_{0.1}^{1}, h_{0.2}^{2}\right\}, e_{2}=\left\{h_{0.2}^{1}, h_{0.3}^{2}\right\}\right\},\right. \\
& \left.\cdot\left\{e_{1}=\left\{h_{0.2}^{1}, h_{0.2}^{2}\right\}, e_{2}=\left\{h_{0.2}^{1}, h_{0.3}^{2}\right\}\right\}\right\}
\end{aligned}
$$

is the fuzzy soft topology of $(\gamma, X)$, for which we get the following conclusions:
Theorem 5. Let $F(U ; E)$ be the whole family of FSSs on $U$ with $E$ as the parameter set. The whole FSS with 
$(\gamma, X) \in F(U ; E)$ and $P(\gamma, X)$ as $(\gamma, X)$ and the subfamily with $\widetilde{\tau}$ as $P(\gamma, X)$; if $\widetilde{\tau}$ is a fuzzy soft topology of $(\gamma, X)$, then $\tilde{\tau}$ is a weak fuzzy soft topology of $(\bar{\gamma}, X)$ and $\tilde{\tau}$ is a strong fuzzy soft topology of $(\bar{\gamma}, X)$.

\section{Proof}

(1) If $\widetilde{\Phi}_{X},(\gamma, X) \in \widetilde{\tau}$, then $\widetilde{\Phi}_{X},(\breve{\gamma}, X) \in \widetilde{\tau}$ and $\widetilde{\Phi}_{X},(\bar{\gamma}$, $X) \in \widehat{\tau}$.

(2) Because $(f, A) \widetilde{c}(g, B)$, so $(\breve{f}, A) \widetilde{c}(\breve{g}, B)$ and $(\widetilde{f}$, A) $\widetilde{c}(\widehat{g}, B)$. If $(f, A),(g, B) \in \widetilde{\tau}$, then $(f, A) \cap(g$, $B) \in \tilde{\tau}$; then, we can get if $(\breve{f}, A),(\breve{g}, B) \in \breve{\tau}$; then, $(\breve{f}, A) \cap_{W}(\breve{g}, B) \in \breve{\tau}$, or if $(\widehat{f}, A),(\bar{g}, B) \in \bar{\tau}$, then $(\bar{f}, A) \cap_{S}(\bar{g}, B) \in \bar{\tau}$.

(3) Because $\left\{\left(f_{k}, A_{k}\right) \mid k \in K\right\} \subset \widetilde{\tau} \Rightarrow \cup_{k \in K}\left(f_{k}, A_{k}\right) \in \tau$, if $\left\{\left(\breve{f}_{k}, A_{k}\right) \mid k \in K\right\} \subset \breve{\tau} \Rightarrow \cup_{W k \in K}\left(\breve{f}_{k}, A_{k}\right) \in \breve{\tau}$ or if $\left\{\left(\hat{f}_{k}, A_{k}\right) \mid k \in K\right\} \subset \bar{\tau} \Rightarrow \cup_{S k \in K}\left(\hat{f}_{k}, A_{k}\right) \in \bar{\tau}$, it can be concluded that Theorem 3 is true.

\section{Conclusion}

In this paper, the intersection and union operation of FSS is extended, and the result of union operation is kept. A series of operation properties of generalized intersection and generalized union of FSSs are given. The fuzzy soft topology based on generalized intersection and generalized union operation of FSS is established because the generalized intersection and generalized union satisfy De Morgan's law. This will provide convenience for the further study of fuzzy soft topological space properties, especially the fuzzy soft quotient topological space. Finally, the topological construction of weak FSS and strong FSS is discussed, and the relationship between them and the topological construction of traditional FSS is obtained.

\section{Data Availability}

The data used to support the findings of this study are available from the corresponding author upon request.

\section{Conflicts of Interest}

The authors declare that they have no conflicts of interest.

\section{Acknowledgments}

This work was supported by key projects of Natural Science Research in Colleges and Universities of Anhui Province (Grant no. KJ2020A0642).

\section{References}

[1] L. A. Zadeh, "Fuzzy sets," Information and Control, vol. 8, no. 3, pp. 338-353, 1965.

[2] K. T. Atanassov, "Intuitionistic fuzzy sets," Fuzzy Sets and Systems, vol. 20, no. 1, pp. 87-96, 1986.
[3] Z. a. Pawlak, "Rough sets," International Journal of Computer \& Information Sciences, vol. 11, no. 5, pp. 341-356, 1982.

[4] D. Molodtsov, "Soft set theory-first results," Computers \& Mathematics with Applications, vol. 37, no. 4-5, pp. 19-31, 1999.

[5] P. K. Maji, A. R. Roy, and R. Biswas, "An application of soft sets in a decision making problem," Computers \& Mathematics With Applications, vol. 44, no. 8-9, pp. 1077-1083, 2002.

[6] N. Cagman and S. Enginoglu, "Soft set theory and uni-int decision making," European Journal of Operational Research, vol. 207, no. 2, pp. 848-855, 2010.

[7] Y. Zou and Z. Xiao, "Data analysis approaches of soft sets under incomplete information," Knowledge-Based Systems, vol. 21, no. 8, pp. 941-945, 2008.

[8] H. Aktaş and N. Çağman, "Soft sets and soft groups," Information Sciences, vol. 177, no. 13, pp. 2726-2735, 2007.

[9] H. Liu Heng, S. G. Li Sheng-Gang, Y. G. Sun Ye-Guo, and H. X. Wang Hong-Xing, "Adaptive fuzzy synchronization for uncertain fractional-order chaotic systems with unknown non-symmetrical control gain," Acta Physica Sinica, vol. 64, no. 7, Article ID 070503, 2015.

[10] Q. M. Sun, Z. L. Zhang, and J. Liu, "Soft sets and soft modules," in Proceedings of the Rough Sets and Knowledge Technology, Third International Conference, RSKT 2008, Springer-Verlag, Chengdu, China, May 2008.

[11] H. Liu, S. G. Li, H. X. Wang, and G. J. Li, "Adaptive fuzzy synchronization for a class of fractional-order neural networks," Chinese Physics B, vol. 26, no. 3, pp. 258-267, 2017.

[12] J. Carlos and R. Alcantud, "Some formal relationships among soft sets, fuzzy sets, and their extensions," International Journal of Approximate Reasoning, vol. 68, pp. 45-53, 2016.

[13] B. Tanay and M. B. Kandemir, "Topological structure of fuzzy soft sets," Computers \& Mathematics with Applications, vol. 61, no. 10, pp. 2952-2957, 2011.

[14] G. Liu, "Rough set approaches in knowledge structures," International Journal of Approximate Reasoning, vol. 138, pp. 78-88, 2021.

[15] S. K. Ghosh and A. Ghosh, "A novel intuitionistic fuzzy soft set based colonogram enhancement for polyps localization," International Journal of Imaging Systems and Technology, vol. 31, no. 3, pp. 1486-1502, 2021.

[16] G. Ayten and Y. Cemil, "Mixed fuzzy soft topological spaces," Journal of Science, vol. 31, no. 4, pp. 1166-1177, 2018.

[17] R. Muhammad and T. Syeda, "On bipolar fuzzy soft topology withdecision-makings," Soft Computing, vol. 4, 2020.

[18] J. C. R. Alcantud, "Soft open bases and a novel construction of soft topologies from bases for topologies," Mathematics, vol. 8, no. 5, p. 672, 2020.

[19] E. Y. Ultami and E..S. Haripamyu, "Jenizon .application of intuitionistic fuzzy soft set to topology," Journal of Physics: Conference Series, vol. 37, p. 1940, 2021.

[20] F. B. Hua and Y. P. Wang, "Analysis and research on ooperation of fuzzy soft set," Journal of Jinggangshan University (Natural Science), vol. 38, no. 6, pp. 6-11, 2017.

[21] B. Ahmad and A. Kharal, "On fuzzy soft sets," Advances in Fuzzy Systems, vol. 2009, no. 4-5, pp. 80-95, 2009. 